\title{
A NEW CONSTRUCTION FOR HADAMARD MATRICES ${ }^{1}$
}

\author{
BY L. D. BAUMERT AND MARSHALL HALL, JR. \\ Communicated by J. D. Swift, August 24, 1964
}

An Hadamard matrix $H$ is a square matrix of ones and minus ones whose row (and hence column) vectors are orthogonal. The order $n$ of an Hadamard matrix is necessarily 1,2 or $4 t$ with $t=1,2,3, \cdots$. It has been conjectured that this condition $(n=1,2$ or $4 t)$ also insures the existence of an Hadamard matrix. Constructions have been given for particular values of $n$ and even for various infinite classes of values. While other constructions exist, those given by [1]-[7] exhaust the previously known values of $n$. This paper gives a new construction which yields, among others, the previously unknown value $n=156$, leaving only two undecided values of $n=4 t \leqq 200$ (these are 116 and 188).

An Hadamard matrix is said to be of the Williamson type if it has the structure imposed by Williamson [6], that is

$$
H=\left|\begin{array}{rrrr}
A & B & C & D \\
-B & A & -D & C \\
-C & D & A & -B \\
-D & -C & B & A
\end{array}\right|,
$$

where each of $A, B, C, D$ is a symmetric circulant $t \times t$ matrix. Notice that if a Williamson type matrix exists for $n=4 t$, then an Hadamard matrix (not obviously Williamson) of order $m=12 t$ would exist provided one could find a $12 \times 12$ matrix with the following properties. Each row and column must contain precisely three $\pm A$ 's, three $\pm B$ 's, three $\pm C$ 's, three $\pm D$ 's and the rows must be formally orthogonal (i.e., $A, B, C, D$ are to be considered as independent quantities). We have discovered such a matrix and display it as Figure 1.

Among the known orders of Williamson type matrices [1], [6], only 52 yields a new value of $n$ by this construction. This gives an Hadamard matrix of order 156. For definiteness, the first rows of $A, B, C, D$ for one of the Williamson type Hadamard matrices of order 52 are given (here + means +1 and - stands for -1 ).

${ }^{1}$ This paper presents the results of one phase of research carried out at the Jet Propulsion Laboratory, California Institute of Technology, under contract number NAS 7-100, sponsored by the National Aeronautics and Space Administration. 


$$
\begin{aligned}
& \begin{array}{lllllllllllll}
1 & 2 & 3 & 4 & 5 & 6 & 7 & 8 & 9 & 10 & 11 & 12 & 13
\end{array} \\
& \text { A }+ \text { + - - + - + + - + - + } \\
& \text { B }+---++++++- \\
& \text { C }+++-++-2++ \\
& \text { D }++-+-++++-+-+
\end{aligned}
$$

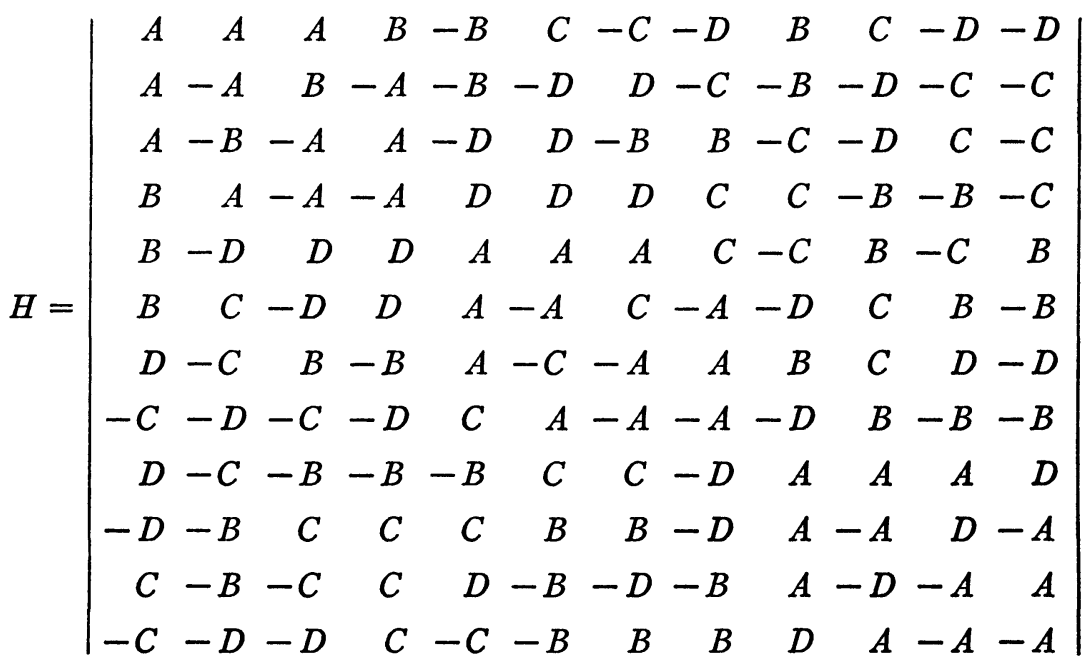

\section{REFERENCES}

1. L. Baumert, S. W. Golomb and M. Hall, Jr., Discovery of an Hadamard matrix of order 92, Bull. Amer. Math. Soc. 68 (1962), 237-238. 225.

2. A. Brauer, On a new class of Hadamard determinants, Math. Z. 58 (1953), 219-

3. K. Goldberg, Hadamard matrices of order cube plus one, Abstract 567-90, Notices Amer. Math. Soc. 7 (1960), 348.

4. R. E. A. C. Paley, On orthogonal matrices, J. Math. and Phys. 12 (1933), 311320.

5. R. G. Stanton and D. A. Sprott, A family of difference sets, Canad. J. Math. 10 (1958), 73-77.

6. J. Williamson, Hadamard's determinant theorem and the sum of four squares, Duke Math. J. 11 (1944), 65-81.

7. - Note on Hadamard's determinant theorem, Bull. Amer. Math. Soc. 53 (1947), 608-613.

Jet Propulsion laboratory, California institute of Technology 